\title{
CD30-Antikörper-Konjugat schafft Durchbruch
}

\author{
Mit Brentuximab Vedotin wurde \\ die erste Substanz seit 1977 für \\ die Behandlung des Hodgkin- \\ Lymphoms (HL) zugelassen.
}

Das Antikörper-Chemotherapie-Konjugat Brentuximab Vedotin erzielte in einer Phase-II-Studie mit 102 Patienten mit primärem oder nach der letzten Vortherapie refraktärem HL bei $75 \%$ der Patienten ein Ansprechen, bei 34\% sogar eine Komplettremission [Younes A et al. J Clin Oncol. 2012;30(18):2183-9]. Dabei dauerte das Ansprechen im Median 6,7 Monate an, die Komplettremission 20,5 Monate und das progressionsfreie Überleben (PFS) lag bei komplettem Ansprechen bei 21,7 Mona- ten. Am beeindruckendsten fand Peter Borchmann, Köln, dass das PFS deutlich länger war als unter der Vortherapie.

Dieser ungewöhnliche Effekt zeigte sich auch in einer Phase-II-Studie zur Wirksamkeit und Sicherheit von Brentuximab Vedotin beim anaplastischen großzelligen Non-Hodgkin-Lymphom (NHL), berichtete Georg Hopfinger, Salzburg, Österreich [Pro B et al. J Clin Oncol. 2012;30(18): 2190-6]. Von 58 Patienten (72\% ALK-negativ, also mit schlechter Prognose) sprachen wiederum $86 \%$ an, davon $57 \%$ komplett.

Die Studie belegt auch, dass der prognostische Nachteil bei ALK-negativen Patienten bei dieser Therapie nicht zu beob- achten ist. Das PFS lag bei 14,3 Monaten, in der letzten Vortherapie war nur ein PFS von 5,9 Monaten erzielt worden.

Als unerwünschte Ereignisse traten bei der Therapie vor allem gut beherrschbare Neutropenien, nach Absetzen der Medikation teils vollständig reversible periphere Neuropathien, Pyrexie, Fatigue oder Diarrhöen auf. In Deutschland ist Brentuximab Vendotin (Adcetris ${ }^{\varpi}$ ) seit 1. Dezember 2012 zugelassen.

Friederike Klein

Satellitensymposium „Brentuximab Vedotin - Eine neue zielgerichtete Therapieoption bei Hodgkin Lymphom und aggressiven T-Zell-Lymphomen" im Rahmen der DGHO-Jahrestagung, Stuttgart, 20.10.2012; Veranstalter: Takeda

\section{Fortgeschrittenes Ovarialkarzinom}

\section{Angiogeneseinhibitor beim platinsensitiven Rezidiv zugelassen}

\author{
Mit der Zulassungserweiterung \\ von Bevacizumab für die Behand- \\ lung des platinsensitiven Ovarial- \\ karzinomrezidivs können nun auch \\ Patientinnen von der Angiogene- \\ sehemmung profitieren, die in der \\ Primärtherapie keinen VEGF-Inhibi- \\ tor erhalten haben.
}

In der Phase-III-Studie OCEANS lebten Ovarialkarzinom-Patientinnen mit platinsensitivem Rezidiv signifikant länger progressionsfrei (PFS), wenn Bevacizumab (Avastin`) zusätzlich zur Standardtherapie Carboplatin/Gemcitabin gegeben wurde (medianes PFS: 12,4 vs. 8,4 Monate; HR 0,484; $\mathrm{p}<0,0001)$.

Entscheidend für eine möglichst lange Überlebenszeit beim fortgeschrittenen Ovarialkarzinom ist die Kombination von State-of-the-Art-Operation und State-ofthe-Art-Chemotherapie, so die Empfehlungen der Arbeitsgemeinschaft Gynäkologische Onkologie (AGO) OVAR. Die zusätzliche Behandlung mit Bevacizumab kann in der Primärtherapie des Ovarialkarzinoms FIGO Stadium IIIB-IV erwogen werden. Sven Mahner, Hamburg-Eppendorf, verwies auf das onkologische Prinzip, die wirksamsten Substanzen so früh wie mög- lich einzusetzen. Bei Patientinnen mit schlechterer Prognose und in späteren Therapielinien scheine, so Mahner, die Wirkung der Chemotherapie nachzulassen, wohingegen Bevacizumab weiterhin aktiv bleibe. Dies sei aber kein Grund, Bevaci- zumab aufgrund der erweiterten Zulassung für spätere Linien „aufzuheben“. Ine Schmale

Pressekonferenz „Avastin ${ }^{\circledast}$ beim Ovarialkarzinom - Zulassungserweiterung für die Rezidivtherapie“, Frankfurt, 27.11.2012; Veranstalter: Roche

mRCC-Zweitlinie mit VEGFR-Inhibitor

\section{Fortgeschrittenes Nierenzellkarzinom}

\section{Axitinib ermöglicht eine Fort- setzung der Behandlung eines fortgeschrittenen Nierenzellkarzi- noms (mRCC) nach Therapie mit Sunitinib oder einem Zytokin.}

Seit September 2012 ist Axitinib (Inlyta ${ }^{\circledR}$ ) zur Behandlung des mRCC bei erwachsenen Patienten nach Versagen einer vorangegangenen Therapie mit Sunitinib oder einem Zytokin zugelassen. Bei Axitinib handelt es sich um einen hochselektiven Inhibitor der VEGF (Vascular Endothelial Growth Factor)-Rezeptoren 1, 2 und 3, die an Tumorwachstum, Neoangiogenese und Metastasenbildung beteiligt sein können. Das Potenzial der Substanz konnte in der Zulassungsstudie AXIS (AXItinib versus Sorafenib in advanced renal cell carcinoma) gezeigt werden. Beim medianen progressionsfreien Überleben führte Axitinib in der Gesamtpopulation im Vergleich zu Sorafenib zu einer signifikanten Verlängerung um mehr als zwei Monate (6,8 vs. 4,7 Monate; HR 0,67; p < 0,0001). Die objektive Ansprechrate betrug unter Axitinib in der Gesamtpopulation 19,4 vs. 9,4\% unter Sorafenib (HR 2,06; $\mathrm{p}=0,0001$ ) [Rini BI et al. Lancet. 2011;378(9807):1931-9]. red

Nach Informationen von Pfizer Pharma 